\title{
Performance of Side Sensitive Group Runs Chart Based on Exact and Random Process Shifts
}

\author{
You Huay Woon \\ Universiti Kebangsaan \\ Malaysia
}

\author{
Michael Khoo Boon Chong \\ Universiti Sains Malaysia
}

\author{
Chong Zhi Lin \\ Universiti Tunku \\ Abdul Rahman
}

\begin{abstract}
A control chart is a crucial statistical tool that is typically used to monitor a process, in an effort to reduce variation. It is necessary to practically evaluate the overall performance of a control chart. Evaluation reveals the efficiency of a control chart to detect a process shift. This influences practitioners' decisions on the choice of a control chart. The usual practice taken to evaluate the performance of a control chart is to use the average run length (ARL). The assumption for using the ARL as a performance measure is that the shift size is known in advance. Determining the shift size can be restrictive, particularly in the case practitioners do not have prior knowledge about the process. In view of this, the expected ARL (EARL) is employed as a performance measure for the random process mean shift. In this article, the overall performance of the side sensitive group runs (SSGR) chart is investigated in terms of EARL. Moreover, the optimal design for the SSGR chart based on minimising the EARL is proposed.
\end{abstract}

Keywords: side sensitive group runs chart, expected average run length, optimal design.

\section{Introduction}

Statistical Process Control (SPC) is a collection of statistical tools that is efficient in achieving stability and improving the capability of a process. A control chart is among the primary tools of SPC (Montgomery 2013). The objective of using a control chart is to monitor the process, and produce high quality output (Steland 2006).

The first control chart was developed by Dr. Walter A. Shewhart, and is referred to as the Shewhart chart. It is well-known that the Shewhart chart is good in detecting large shifts, but rather insensitive to detect small mean shifts. In view of this, new control charts such as the group runs (GR) chart (Gadre and Rattihalli 2004), and side sensitive group runs (SSGR) chart (Gadre and Rattihalli 2007), are developed to enhance the detection ability toward small and moderate sized process shifts.

The performance of the control chart influences the decision on the choice of a control chart. It is thus crucial to evaluate the performance of a control chart. The average run length (ARL) is the common performance measure for a control chart. The ARL represents the average number of samples plotted on a control chart, before an out-of-control signal is detected. 
The computation of the ARL requires the practitioners to specify the magnitude of shift (Chakraborti 2007).

In reality, this requirement is too restrictive, since the practitioners may not have historical knowledge about the process. Therefore, practitioners may not be able to determine the precise shift size of the process in advance. In this circumstance, expected ARL (EARL) is employed, where the EARL does not require practitioners to identify the shift size. On the contrary, the EARL computes the expected value of the ARL, over the distribution function of the shift size. Celano (2009), Castagliola, Celano, and Psarakis (2011) and You, Khoo, Castagliola, and $\mathrm{Qu}(2016)$ evaluated the performance of a control chart when the shift size is unknown.

Gadre and Rattihalli (2007) proposed the SSGR based on average time to signal (ATS), which is ATS $=n \times$ ARL i.e. $n$ denotes sample size. However, the SSGR chart uses the fixed $n$ in process monitoring. Therefore, measuring the SSGR chart's performance using either the ATS or ARL gives a similar conclusion. In addition, Gadre and Rattihalli (2007) demonstrated that the performance of the SSGR chart is superior to the Shewhart chart, synthetic chart and GR chart, and this has motivated the current research work.

This paper aims to propose the optimal design for the SSGR chart based on minimising EARL. Moreover, the performance of the SSGR chart based on exact and random process shift sizes is investigated. The SSGR chart is briefly reviewed in the following section. Next, the run length properties of the SSGR chart are discussed in Section 3. The optimal design of the SSGR chart based on minimising the out-of-control EARL is presented in Section 4. This is followed by the investigation of the performance of the SSGR chart based on the exact and random shifts for monitoring the process mean. Finally, some concluding remarks are presented.

\section{The SSGR chart}

The SSGR chart is the integration of the Shewhart sub-chart and an extended version of the conforming run length (CRL) sub-chart. In process monitoring, take a sample of $n$ observations and compute the sample value, i.e., $\bar{X}$. When a sample value plots beyond the control limits of the Shewhart sub-chart, the SSGR chart does not signal an out-of-control status. Alternatively, it shows that a nonconforming sample exists. Further investigation using CRL sub-chart is required to determine the state of the process.

As the name implies, the SSGR chart takes into account the side sensitivity aspects, i.e. the two continuous sample values that fall beyond the control limits of the Shewhart sub-chart must be on the same side of the Shewhart sub-chart. Therefore, the SSGR chart declares an out-of-control if (i) $\mathrm{CRL}_{1} \leq L$ or (ii) $\mathrm{CRL}_{r} \leq L$ and $\mathrm{CRL}_{r+1} \leq L$, for $r=2,3, \ldots$, and that both $\mathrm{CRL}_{r}$ and $\mathrm{CRL}_{r+1}$ have shifts on the same side of the Shewhart sub-chart. Here, $L$ is the lower limit of the CRL sub-chart, which must be presented as a positive value. The $\mathrm{CRL}_{r}$, for $r=1,2, \ldots$ is the number of conforming samples plotted on the Shewhart sub-chart, before a nonconforming sample is detected. With an additional rule to enhance the detection effectiveness, the SSGR chart is considered in this work.

\section{The run length properties of the SSGR chart}

When process parameters, i.e. the in-control mean $\mu_{0}$ and in-control standard deviation $\sigma_{0}$ are known, the control limits of the Shewhart sub-chart are defined as follows:

$$
L C L=\mu_{0}-\frac{K}{\sqrt{n}} \sigma_{0}
$$

and 


$$
U C L=\mu_{0}+\frac{K}{\sqrt{n}} \sigma_{0}
$$

where the $L C L, U C L$ and $K$ represent the lower control limit, upper control limit and control limit coefficient respectively. Note that $n$ denotes the sample size.

The probability that a sample is nonconforming on the Shewhart sub-chart is defined as $A=$ 1 - $\operatorname{Pr}(\bar{X} \in[L C L, U C L])$, i.e.

$$
A=1-\operatorname{Pr}\left(\mu_{0}-\frac{K}{\sqrt{n}} \sigma_{0} \leq \bar{X} \leq \mu_{0}+\frac{K}{\sqrt{n}} \sigma_{0}\right) .
$$

Using the fact that $\bar{X} \sim N\left(\mu_{0}+\delta \sigma_{0}, \sigma_{0}^{2} / n\right)$, subtracting $\mu_{0}+\delta \sigma_{0}$ and multiplying the inequality by $\sqrt{n} / \sigma_{0}$, Equation (3) becomes

$$
A=1-\left[\Phi\left[\left(\mu_{0}+\frac{K}{\sqrt{n}} \sigma_{0}-\mu_{0}-\delta \sigma_{0}\right) \frac{\sqrt{n}}{\sigma_{0}}\right]-\Phi\left[\left(\mu_{0}-\frac{K}{\sqrt{n}} \sigma_{0}-\mu_{0}-\delta \sigma_{0}\right) \frac{\sqrt{n}}{\sigma_{0}}\right]\right] .
$$

Next, following some simplifications, $A$ is reduced to

$$
A=1-\Phi(K-\delta \sqrt{n})+\Phi(-K-\delta \sqrt{n}) .
$$

Here, $\Phi(\cdot)$ is the standard normal cumulative distribution function (cdf).

Moreover, the probability of an event $\mathrm{CRL}_{r} \leq L$ is

$$
P=1-(1-A)^{L}
$$

Furthermore, the conditional probability for taking into account the position where the sample value falls on the Shewhart sub-chart is

$$
\beta=\frac{\operatorname{Pr}\left(\bar{X}>\mu_{0}+\frac{K}{\sqrt{n}} \sigma_{0}\right)}{A}=\frac{1-\Phi(K-\delta \sqrt{n})}{A} .
$$

Note that the reader can refer to Gadre and Rattihalli (2007) for a detailed and complete derivation.

The ARL formula for the SSGR chart is

$$
\mathrm{ARL}=\frac{1-\beta(1-\beta) P^{2}}{A P^{2}[1+\beta(1-\beta)(P-2)]} .
$$

Note that the computation of the ARL can be conducted when the practitioner is able to identify the shift size in the process mean.

However, in real scenarios, the shift size is unknown in advance, as the practitioner does not have historical knowledge of the overall process. In light of this, it is crucial to consider the EARL for an overall range of shifts $\left(\delta_{\min }, \delta_{\max }\right)$, where $\delta_{\min }$ and $\delta_{\max }$ indicate the lower and upper bounds of the mean shift respectively. The EARL of the SSGR chart is denoted as follows

$$
\mathrm{EARL}=\int_{\delta_{\min }}^{\delta_{\max }} f_{\delta}(\delta) \mathrm{ARL} d \delta,
$$

where $f_{\delta}(\delta)$ is the probability density function of the magnitude of the shift in a process and ARL is from Equation (8). The magnitude is denoted as $\delta$. 


\section{Optimal design of the SSGR chart based on minimising EARL}

In many scenarios, the magnitude of shift size, $\delta$ is seldom predefined. When the real shift size differs from the desired $\delta$, optimal charting parameters based on minimising out-of-control $\operatorname{ARL}\left(\mathrm{ARL}_{1}\right)$ result in a deterioration in performance. For example, in Table 2, when $n=$ 3 and $\delta=0.8$, the optimal charting parameters $(K, L)=(1.9588,7)$ give $\mathrm{ARL}_{1}=4.32$. However, if the real mean shift occurred in the process is $\delta=0.2$, using the same optimal charting parameters $(K, L)$ will result in $\mathrm{ARL}_{1}=143.88$. Comparing this $\mathrm{ARL}_{1}$ value with the minimized $\mathrm{ARL}_{1}=127.88$ (refer Table 2 when $(n, \delta)=(3,0.2)$ ), the relative error is 100 $\mathrm{x}(143.88-127.88) / 127.88=12.51 \%$.

In light of this, it is crucial to consider the EARL by taking into account the overall range of shifts $\left(\delta_{\min }, \delta_{\max }\right)$. The computation of the optimal charting parameters $(K, L)$ that minimize the out-of-control EARL $\left(\mathrm{EARL}_{1}\right)$, for the SSGR chart is

$$
(K, L)=\arg \min \operatorname{EARL}\left(n, K^{\prime}, L^{\prime}, \delta_{\min } \delta_{\max }\right)
$$

subject to the constraint EARL $\left(n, K^{\prime}, L^{\prime}, 0,0\right)=\mathrm{EARL}_{0}$, where $\mathrm{EARL}_{0}$ is the in-control EARL. Here, $\mathrm{EARL}_{1}\left(n, K^{\prime}, L^{\prime}, \delta_{\min }, \delta_{\max }\right)$ is obtained using Equation (9).

A computer program developed in ScicosLab software version 4.4.2 (www.scicoslab.org) was employed to compute the optimal charting parameters $(K, L)$ based on the following steps:

Step 1: Specify $n,\left(\delta_{\min }, \delta_{\max }\right), \mathrm{EARL}_{0}$.

Step 2: Initialize $L$ as unity.

Step 3: Compute $K$ using a nonlinear equations solver to satisfy $\mathrm{EARL}_{0}$ that specified in Step (1).

Step 4: Compute the EARL 1 using Equation (9), based on the current charting parameters $(K, L)$.

Step 5: Increase $L$ by one if $L=1$ or " $L>1$ and EARL $L_{1}$ has been reduced". Otherwise, proceed to the next step.

Step 6: Take the charting parameters $(K, L)$ that give the smallest $\mathrm{EARL}_{1}$ as the optimal charting parameters.

\section{The performance comparison of the SSGR chart}

Table 1 presents the optimal charting parameters $(K, L)$ and the corresponding out-of-control EARL $\left(\mathrm{EARL}_{1}\right)$ for various $\left(n, \delta_{\min }, \delta_{\max }\right)$ combinations. In addition, the optimal charting parameters $(K, L)$ are adjusted to give the intended in-control EARL $\left(\mathrm{EARL}_{0}\right)$ at 370.4. Here, the SSGR chart is optimally designed to obtain the optimal charting parameters $(K$, $L$ ) based on the procedure illustrated in Section 4. For example, when $n=5, \delta_{\min }=0.2$ and $\delta_{\max }=1.0$, the optimal pairs $(K, L)=(2.1735,16)$ yield the smallest $\mathrm{EARL}_{1}$, i.e. 13.18 and attaining the $\mathrm{EARL}_{0}$ at 370.4 . 
Table 1: $\mathrm{EARL}_{1} \mathrm{~s}$ for different combinations of $\left(n, \delta_{\min }, \delta_{\max }\right)$, based on the optimal charting parameters $(K, L)$.

\begin{tabular}{lllll}
\hline$n$ & $\delta_{\min }$ & $\delta_{\max }$ & $(K, L)$ & $\mathrm{EARL}_{1} \mathrm{~S}$ \\
\hline 3 & 0.2 & 1.0 & $(2.2284,20)$ & 23.84 \\
& 1.0 & 2.0 & $(1.7185,3)$ & 1.41 \\
5 & 0.2 & 1.0 & $(2.1735,16)$ & 13.18 \\
& 1.0 & 2.0 & $(1.5953,2)$ & 1.11 \\
7 & 0.2 & 1.0 & $(2.1213,13)$ & 8.70 \\
& 1.0 & 2.0 & $(1.5953,2)$ & 1.04 \\
9 & 0.2 & 1.0 & $(2.0785,11)$ & 6.34 \\
& 1.0 & 2.0 & $(1.5953,2)$ & 1.01 \\
\hline
\end{tabular}

In order to illustrate the implementation of the proposed optimal design, Table 2 displays the optimal charting parameters based on minimising out-of-control ARL $\left(\mathrm{ARL}_{1}\right)$ and the corresponding $\mathrm{ARL}_{1} \mathrm{~s}$ for the same $n$ values. For the sake of comparison, $\delta \in\{0.2,0.4,0.8,1.2,1.4,2.0\}$ are considered. Here, $\delta \in\{0.2,0.4,0.8\}$ and $\delta \in\{1.2,1.4,2.0\}$ are included in $\left(\delta_{\min }, \delta_{\max }\right)=$ $(0.2,1.0)$ and $\left(\delta_{\min }, \delta_{\max }\right)=(1.0,2.0)$, respectively. In Table 2 , when $n=3$ and $\delta=1.2$, the $(K, L)$ that minimize $\mathrm{ARL}_{1}$ are $(1.7185,3)$. These optimal charting parameters yield the smallest $\mathrm{ARL}_{1}$ value, i.e. $\mathrm{ARL}_{1}=1.72$ while attaining the desired $\mathrm{ARL}_{0}$ at 370.4.

Table 2: $\mathrm{ARL}_{1} \mathrm{~s}$ for different combinations of $(n, \delta)$, based on the optimal charting parameters $(K, L)$.

\begin{tabular}{llll}
\hline$n$ & $\delta$ & $(K, L)$ & $\mathrm{ARL}_{1} \mathrm{~S}$ \\
\hline 3 & 0.2 & $(2.4125,44)$ & 127.88 \\
& 0.4 & $(2.2403,21)$ & 29.55 \\
& 0.8 & $(1.9588,7)$ & 4.32 \\
& 1.2 & $(1.7185,3)$ & 1.72 \\
& 1.4 & $(1.7185,3)$ & 1.35 \\
& 2.0 & $(1.5953,2)$ & 1.03 \\
5 & 0.2 & $(2.3606,35)$ & 81.44 \\
& 0.4 & $(2.1401,14)$ & 14.72 \\
& 0.8 & $(1.8025,4)$ & 2.31 \\
& 1.2 & $(1.5953,2)$ & 1.21 \\
& 1.4 & $(1.5953,2)$ & 1.07 \\
& 2.0 & $(1.5953,2)$ & 1.00 \\
7 & 0.2 & $(2.3171,29)$ & 57.05 \\
& 0.4 & $(2.0785,11)$ & 9.13 \\
& 0.8 & $(1.7185,3)$ & 1.66 \\
& 1.2 & $(1.5953,2)$ & 1.07 \\
& 1.4 & $(1.5953,2)$ & 1.02 \\
& 2.0 & $(1.5953,2)$ & 1.00 \\
9 & 0.2 & $(2.2821,25)$ & 42.50 \\
& 0.4 & $(2.0260,9)$ & 6.40 \\
& 0.8 & $(1.7185,3)$ & 1.37 \\
& 1.2 & $(1.5953,2)$ & 1.02 \\
& 1.4 & $(1.5953,2)$ & 1.00 \\
& 2.0 & $(1.5953,2)$ & 1.00 \\
\hline
\end{tabular}

According to Table 1, EARL $\mathrm{E}_{1}=6.34$ is obtained using the optimal charting parameters $(K, L)=(2.0785,11)$ when $n=9, \delta_{\min }=0.2$ and $\delta_{\max }=1.0$. By considering $\delta=0.4$ (i.e. $\left.\delta \in\left(\delta_{\min }, \delta_{\max }\right)\right)$ for the same $n$ value, the $\mathrm{ARL}_{1}$ value is 6.46 using the same optimal charting parameters. This value is almost the same as the $\mathrm{ARL}_{1}=6.40$ when using the optimal charting parameters $(K, L)=(2.0260,9)$ from Table 2 . This shows that the value of the performance measures, i.e. $\mathrm{EARL}_{1}$ and $\mathrm{ARL}_{1}$ are almost the same. Thus, the optimal 
charting parameters computed using minimising EARL $\mathrm{E}_{1}$ can be implemented as long as $\delta \in$ $\left(\delta_{\min }, \delta_{\max }\right)$, i.e. when the practitioners do not have prior knowledge to determine the exact process shift size.

\section{Conclusion}

This paper assessed the performance of the SSGR chart based on ARL and EARL when the magnitude of shift is deterministic, and the magnitude of the shift may not be known in advance respectively. Here, the ARL is used when the next shift size is known in advance. In a real-world application, there is the situation during which practitioners cannot specify the magnitude of shift in advance. In this scenario, if a chart's user considers a certain shift size and uses the corresponding optimal charting parameters, the performance of the SSGR chart will significantly deteriorate if a different shift size occurs in reality. Therefore, EARL is implemented to evaluate the SSGR chart when dealing with random shifts. The results reveal that adopting the optimal charting parameters based on minimising EARL $_{1}$ is reliable, as the performance measure is nearly the same when using the optimal charting parameters based on minimising $\mathrm{ARL}_{1}$. In view of this, the proposed optimal charting parameters can be implemented as long as $\delta \in\left(\delta_{\min }, \delta_{\max }\right)$. Future research works can consider to propose the optimal design of the SSGR chart based on minimizing EARL when the process parameters are estimated.

\section{Acknowledgement}

This research is supported by the Universiti Kebangsaan Malaysia, Geran Galakan Penyelidik Muda, GGPM-2017-062.

\section{References}

Castagliola P, Celano G, Psarakis S (2011). "Monitoring the Coefficient of Variation Using EWMA Charts." Journal of Quality Technology, 43(3), 249-265. doi:https://doi.org/ 10.1080/00224065.2011.11917861.

Celano G (2009). "Robust Design of Adaptive Control Charts for Manual Manufacturing/Inspection Workstations." Journal of Applied Statistics, 36(2), 181-203. doi:https: //doi.org/10.1080/02664760802443947.

Chakraborti S (2007). "Run Length Distribution and Percentiles: The Shewhart $\bar{X}$ Chart with Unknown Parameters." Quality Engineering, 19(2), 119-127. doi:https://doi.org/ $10.1080 / 08982110701276653$.

Gadre MP, Rattihalli RN (2004). "A Group Runs Control Chart for Detecting Shifts in the Process Mean." Economic Quality Control, 19(1), 29-43. doi:https://doi.org/10.1515/ EQC. 2004.29.

Gadre MP, Rattihalli RN (2007). "A Side Sensitive Group Runs Control Chart for Detecting Shifts in the Process Mean." Statistical Methods and Applications, 16(1), 27-37. doi: DOI10.1007/s10260-006-0013-9.

Montgomery DC (2013). Introduction to Statistical Quality Control. New York: John Wiley \& Sons, Inc.

Steland A (2006). "A Bootstrap View on Dickey-Fuller Control Charts for AR(1) Series." Austrian Journal of Statistics, 35(2 \& 3), 339-346. doi:https://doi.org/10.17713/ ajs.v35i2\&3.381. 
You HW, Khoo MBC, Castagliola P, Qu L (2016). "Optimal Exponentially Weighted Moving Average Charts with Estimated Parameters based on Median Run Length and Expected Median Run Length." International Journal of Production Research, 54(17), 5073-5094. doi:https://doi.org/10.1080/00207543.2016.1145820.

\section{Affiliation:}

You Huay Woon

Pusat PERMATApintar Negara

Universiti Kebangsaan Malaysia

43600 UKM Bangi, Selangor, Malaysia

E-mail: hwyou@ukm.edu.my

\section{Austrian Journal of Statistics}

published by the Austrian Society of Statistics

Volume 47

June 2018 http://www .ajs.or.at/

http://www.osg.or.at/

Submitted: 2018-04-29

Accepted: 2018-05-25 